\title{
Bioremediation of melanoidin contamination in distillery effluent using Aspergillus brasiliensis
}

\author{
Tanim ArPit Singh ${ }^{1 *}$, Trashi Singh ${ }^{2}$, Ranjan Singh ${ }^{3}$, Prabhash Kumar Pandey ${ }^{4}$,

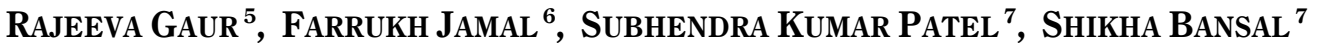 \\ ${ }^{1}$ Maharaja Ranjit Singh College of Professional Sciences, Indore (M.P.), India \\ ${ }^{2}$ Cyanobacterial Research Lab, Rani Durgavati University, Jabalpur (M.P.), India \\ ${ }^{3}$ Choithram College of Professional Studies, Indore (M.P.), India \\ ${ }^{4}$ Department of Biochemistry, Allahabad University, Allahabad (U.P.), India \\ ${ }^{5}$ Department of Microbiology, RamManohar Lohia Avadh University, Ayodhya (U.P.), India \\ ${ }^{6}$ Department of Biochemistry, RamManohar Lohia Avadh University, Ayodhya (U.P.), India \\ ${ }^{7}$ Department of Microbiology, St. Aloysius College Autonomous, Jabalpur (M.P.), India
}

\begin{abstract}
The current investigation is the first report of utilization of Aspergillus brasiliensis for the decolorization of melanoidin in distillery effluent. The effluent generated from alcohol distilleries is one of the most complex wastewater with a high biological oxygen demand (BOD) and other organic, inorganic, and toxic constituents. The effluent contains melanoidin, a dark brown compound, which is difficult to remediate by using conventional technologies. The disposal of spent wash in the natural environment is hazardous and can deteriorate land and water resources. The decolorization of spent wash through physical and chemical methods remains unsuitable, and the only alternative to decolorize spent wash is biological treatment. In the current study, three fungal strains were isolated from the distillery waste and screened for their ability to decolorize melanoidin. The isolate RS2 exhibited maximum decolorization of $83 \%$ and was identified as Aspergillus brasiliensis. Its optimum growth temperature was $37^{\circ} \mathrm{C}$, and the maximum efficiency was recorded after $120 \mathrm{~h}$ of incubation. Nutritional sources were investigated for the fungi showing the maximum decolorization of melanoidin, and starch and peptone were found to be the best carbon and nitrogen sources, respectively. At $1.5 \%$ starch concentration and $1.5 \%$ peptone concentration, the decolorization level attained was $87.45 \%$ and $88.74 \%$, respectively. A. brasiliensis exhibited a high potential to decolorize melanoidin. The decolorization percentage was high, which makes this fungus a potential candidate for use at the industrial scale for the bioremediation of spent wash.
\end{abstract}

Key words: spent wash; effluent; melanoidin; bioremediation; decolorization

\section{Introduction}

Alcohol distilleries are considered to be one of the most polluting industries as they generate large amounts of effluent rich in pollutants. In India, there are more than 500 distilleries, and they generate 2.75 billion liters of alcohol and produce 50 billion liters of effluent per year (Subramanian et al., 2005; Tiwari et al., 2007). The majority of distilleries are attached to sugar factories, and their effluent is known as spent wash. For every one liter of alcohol produced, 10-15 liters of spent wash is generated. If the daily generation of spent wash is cal- culated, in a general distillery where ethanol is produced from cane molasses, half a million liter of spent wash is generated daily (Tiwari et al., 2012). Alcohol distillery tops the list in the Red Category industries, with a high polluting potential, according to the Ministry of Environment and Forest (MOEF), the Government of India (Tiwari et al., 2007).

The spent wash from distilleries is not only high in organic and inorganic contaminants but also contains dark brown recalcitrant compounds known as melanoidin. These natural compounds are formed by the con-

\footnotetext{
${ }^{*}$ Corresponding author: Maharaja Ranjit Singh College of Professional Sciences, Indore (M.P.), India; e-mail: tanim.arpit@gmail.com
} 
densation of sugars and amino acids by a non enzymatic activity (Sharma et al., 2018). The distillery effluents containing melanoidin cause serious soil and water pollution when disposed. Their major negative effects include eutrophication in aquatic environments and reduction of sunlight penetration in rivers, lakes, and lagoons, which in turn, decrease both the photosynthetic activity and the dissolved oxygen concentrations, thereby affecting the normal life cycle of aquatic fauna and flora due to the formation of anaerobic environment (Bernardo et al., 1997). When discharged in open lands, melanoidin reduces soil alkalinity and causes manganese deficiency in soil, which inhibits seed germination and damages vegetation (Tiwari et al., 2012).

A cost-effective and efficient melanoidin treatment method is yet to be established. Melanoidin is highly resistant to degradation by microorganisms as its antioxidant properties are toxic to them (Fito et al., 2019). Conventional treatment methods include physiochemical treatment technologies such as adsorption performed using activated charcoal. Other treatment technologies include flocculation or coagulation of melanoidin using $\mathrm{FeCl}_{3}$ or $\mathrm{AlCl}_{3}$. All these conventional methods, however, proved to be ineffective. Moreover, the color can even intensify during the anaerobic treatment due to re-polymerization of compounds (Chandra et al., 1997). The disposal of spent wash even after conventional treatment remains hazardous and has a high pollution potential due to the accumulation of non biodegradable recalcitrant melanoidin compounds (Tiwari et al., 2012).

The decolorization of melanoidin compounds through microbial degradation has the potential to become an ecofriendly and cost-efficient alternative to the current methods of remediation of spent wash (Kumar et al., 1998; Moosvi et al., 2005; Naik et al., 2010). This is a good alternative to chemical treatment as an effective removal of melanoidin can be achieved using microorganisms (Karnwal, 2019). In the past decade, melanoidin degradation by bacteria and actinobacteria has received significant attention (Mohana et al., 2007). Several other microorganisms such as yeast were also used for decolorization of melanoidin (Raghukumar and Rivonkar 2001). The potential of fungi such as Phanerochaete chrysosporium, Aspergillus sp., P. pinophilum, and Emercella nidulans has also been tested for the treatment of distillery spent wash (Pant and Adholeya, 2007).
Microorganisms are ubiquitously distributed in the environment, and therefore, the isolation and screening for potentially decolorizing microorganisms of interest can be easily performed from natural ecosystems. In the present study, an attempt was made to isolate fungus from spent wash-polluted soil and distillery effluent and to test its ability to decolorize melanoidin.

\section{Materials and methods}

\section{Collection of distillery effluent samples}

Three samples of molasses wastewater were collected in sterile polyethylene bags (spent wash/distillery effluent) from the adjoining areas of Bajaj Hindustan Sugar Pvt. Ltd. Khambharkhera, Lakhimpur Kheri (U.P.), India, by a random sampling method. All the samples were stored at $4{ }^{\circ} \mathrm{C}$ before the analysis. The collected samples were characterized for $\mathrm{pH}$, odor, and color.

\section{Isolation of fungal strains degrading melanoidin}

The fungi were isolated from molasses wastewater by a dilution plating method. One milliliter of the effluent sample was dispersed thoroughly in $10 \mathrm{ml}$ of sterile distilled water, thus making $1 / 10$ dilution of the solution. From this sample solution, $1 \mathrm{ml}$ was transferred to $9 \mathrm{ml}$ of distilled water. The solution thus obtained had the concentration of $1 / 100$ or $10^{-2}$ dilutions. From the diluted sample, $1 \mathrm{ml}$ was transferred into sterile petri dishes containing potato dextrose agar (PDA) supplemented with streptomycin $(3 \mu \mathrm{g} / \mathrm{ml})$. The plates were incubated at $28^{\circ} \mathrm{C}$ for 7 days, following which fungal colonies appearing on PDA were isolated and preserved at $4^{\circ} \mathrm{C}$. The fungal colonies were identified according to their morphological appearance. The colonies were large with a velvety thick texture and a radially furrowed zonation on the reverse side.

\section{Primary screening for dye decolorization ability of fungal strains}

In preliminary screening experiments, solid-plate decolorization was performed (Vasudev and Kuhad, 1994). PDA was incorporated with bromophenol blue at a concentration of $10 \mathrm{mg} / \mathrm{l}$, and the plates were incubated with mycelia plug ( $8 \mathrm{~mm}$ diameter each) taken from the periphery of an actively growing fungal colony. Incubation was performed at $25 \pm 2^{\circ} \mathrm{C}$ in dark till the edge of a single colony almost touched theplate wall. Decoloriza- 
tion in a solid medium was observed by visual disappearance of color. Decolorization zones or diffusion spots were observed when the fungi degraded the dye (bromophenol blue). The fungi that completely degraded the dye by utilizing it as a nutritional source were chosen for further studies.

\section{Secondary screening for melanoidin decolorization potential}

The quantitative removal/decolorization of melanoidin was estimated bya decolorization assay. The effluent was first centrifuged at $5600 \mathrm{~g}$ for $10 \mathrm{~min}$ and then filtered through Whatman No. 1 filter paper (HiMedia, India). The effluent was inoculated with the isolated fungal strains and incubated at $37^{\circ} \mathrm{C}$. After the submerged fermentation process was terminated, the optical density of the spent wash was recorded using a UV-visible spectrophotometer at $475 \mathrm{~nm}$. The percentage of decolorization was calculated by measuring the decrease in the absorbance at $475 \mathrm{~nm}$ against the initial absorbance value.

\section{Inoculum preparation}

The cell suspension was prepared by inoculating $1 \mathrm{ml}$ of $24 \mathrm{~h}$ grown culture to $50 \mathrm{ml}$ basal broth and then incubating the medium at $37^{\circ} \mathrm{C}$ for $24 \mathrm{~h}$ to achieve an active exponential phase of the culture consisting of $5 \times 10^{6} \mathrm{cfu} / \mathrm{ml}$. The culture was then transferred into the flask and incubated under static conditions at $37^{\circ} \mathrm{C}$ for $24 \mathrm{~h}$.

\section{Decolorization assay of the distillery effluent}

For the initial screening of melanoidin-degrading fungi, the fungal isolates were inoculated into Erlenmeyer flasks containing $20 \mathrm{ml}$ of $10 \%$ distillery effluents. Glucose $(1 \%)$ was added as a readily available carbon source for the isolates (Tiwari et al., 2012). The flasks were incubated at $37^{\circ} \mathrm{C}$ under aerobic conditions and observed daily for any reduction in the color of the distillery effluent. Samples were withdrawn after every $24 \mathrm{~h}$ to estimate the degree of melanoidin decolorization. For the decolorization assay, the samples were centrifuged at $5600 \mathrm{~g}$ for $10 \mathrm{~min}$. The supernatants of the centrifuged samples were analyzed spectrophotometrically at $475 \mathrm{~nm}$. The decolorization yield was expressed as the decrease in the absorbance at $475 \mathrm{~nm}$ against the initial absorbance value. An uninoculated medium served as control. The assay was performed in triplicates and com- pared with control. The removal/decolorization efficiency of the isolates was expressed as follows:

Decolorization [\%] =

$$
=\frac{\text { Initial absorbance }- \text { Final absorbance }}{\text { Initial absorbance }} \times 100
$$

\section{Identification of the selected fungal isolate}

The selected fungal isolate RS2 exhibited the highest decolorization of melanoidin. The strain was identified at the Centre for Medical Diagnostics and Research (CMDR), Motilal Nehru National Institute of Technology, Allahabad (U.P.), India, by using the BIOLOG method (Frąc, 2012; Pertile et al., 2020).The identification was performed using BIOLOG FF MicroPlate ${ }^{\mathrm{TM}}$, which is designed for rapid identification of filamentous fungi. The FF MicroPlate ${ }^{\mathrm{TM}}$ uses redox chemistry based on the reduction of tetrazolium, which responds to the process of fungal utilization of carbon sourcesand substrates. The system analyzes both the color development and turbidity within the microplate. The Biolog FF MicroPlate $^{\mathrm{TM}}$ performs 95 discrete tests simultaneouslyand gives a characteristic reaction pattern called the "fingerprint". These fingerprint reaction patterns provide a vast amount of information about the metabolic properties of the tested fungus, alongwith its identification up to the species level. These "fingerprints" are matched with the BIOLOG database MicroLog ${ }^{\mathrm{TM}}$, which contains reaction fingerprints of different fungal species.

The FF database also includes a photo library containing macroscopic and microscopic photographs of the fungi. This photolibrary ensures the exact identification of unknown organisms by providing visual and morphological verification of the identified species.

\section{Effect of temperature on the growth of the fungal isolate RS2}

The effect of temperature on the growth of the RS2 isolate was evaluated on the PDA growth medium containing spent wash. The plates were inoculated with a $96 \mathrm{~h}$ old culture of RS2. The inoculated plates were placed in plastic bags and incubated in dark at $5,28,32$, 37 , and $45^{\circ} \mathrm{C}$ (Cetin and Donmez, 2006).

\section{Effect of pH on melanoidin decolorization}

To determine the optimum $\mathrm{pH}$ for the maximum decolorization by the selected RS2 strain, the $\mathrm{pH}$ of the distillery effluent was adjusted to different $\mathrm{pH}$ values (4.0, 
$4.5,5.0,5.5,6.0,6.5$, and 7.0) by using $\mathrm{pH}$ buffer tablets. The effluent samples were inoculated with a $96 \mathrm{~h}$ old culture of the RS2 isolate and incubated at $37^{\circ} \mathrm{C}$. To analyze color reduction, samples were drawn at 2 day intervals up to 11 days post incubation.

\section{Effect of carbon source on melanoidin decolorization}

Different carbon sources were used to promote the growth of the selected fungal isolate RS2 and analyze melanoidin decolorization. One hundred milliliters of 30\% effluent was taken, and the $\mathrm{pH}$ was adjusted to 5 . Different carbon sources such as dextrose, maltose, lactose, starch, and sucrose were tested for their effects on decolorization. They were added to the distillery effluent at $2 \%$ concentration, and the media were inoculated with the selected isolate. The flasks were incubated at $37^{\circ} \mathrm{C}$. All the inoculated samples were drawn at 1 day intervals for upto 7 days to analyze the level of melanoidin reduction.

Initially, when the carbon source was added, the effluent was colored as it contained melanoidin. The fungal isolate RS2 decolorized it, and the percentage of decolorization was determined by measuring the difference in the optical density of the effluent at $425 \mathrm{~nm}$.

\section{Effects of different concentrations of starch on the decolorization potential of the fungal isolate}

To optimize the concentration of carbon source for the maximum decolorization, different concentrations of starch $(0.5,1.0,1.5,2.0,2.5$, and 3.0\%) were added to the distillery effluent, and their effect on the decolorization of melanoidin pigment pollution was recorded.

\section{Effect of nitrogen source on melanoidin decolorization}

Different nitrogen sources such as ammonium sulfate, peptone, yeast extract, sodium nitrate, and beef extract were tested for their effects on the decolorization of the distillery effluent by the selected fungal strain. These nitrogen sources were added at $30 \%$ concentration to the distillery effluent. The carbon source at an optimized concentration was added to all the samples. The percentage of decolorization was determined by measuring the difference in the optical density of the effluent at $425 \mathrm{~nm}$. The best nitrogen source was selected on the basis of the decolorization potential of the fungi.

\section{Effect of different concentrations of peptone on the decolorization potential of the fungi}

To optimize the level of peptone for the maximum decolorization potential, it was added to the distillery effluent at $0.5,1.0,1.5,2.0,2.5$, and $3.0 \%$ concentrations. The effects of different concentrations of peptone on melanoidin decolorization were recorded. The decolorization potential of the fungi was determined by measuring the difference in the optical density at $425 \mathrm{~nm}$.

\section{Statistical analysis}

All the experiments were conducted in triplicates. Standard deviation for each experimental result was calculated using Microsoft Excel.

\section{Results}

\section{Collection of the distillery effluent sample}

The collected distillery effluent samples were dark reddish brown in appearance due to the presence of melanoidin. The effluent had a strong unpleasant odor and an alkaline $\mathrm{pH}$ of 8.7.

\section{Isolation of melanoidin-degrading fungal strains}

Three fungal strains were isolated from the effluent samples. These isolates were named RS1, RS2, and RS3. The fungal strains were maintained and preserved on PDA and were laterscreened for their ability to degradethe dye.

\section{Primary screening for dye decolorization ability of the fungal strains}

The fungal isolates RS1, RS2, and RS3 were analyzed for their ability to decolorize the bromophenol blue dye. The highest degree of decolorization was observed for the RS2 isolate, which yielded a clear zone of $38 \mathrm{~mm}$ after 3 days of inoculation. On the $10^{\text {th }}$ day, the isolate RS2 covered and cleared the entire dye from the plate. The remaining two isolates (RS1 and RS3) degraded the dye, and the observed zones of clearance were 21 and $24 \mathrm{~mm}$, respectively, on the third day of incubation. These isolates were unable to degrade the dye completely even after 10 days of incubation.

\section{Secondary screening for melanoidin decolorization potential}

A quantitative analysis was performed to evaluate the potential of all the three fungal strains to degrade melanoidin. The RS2 isolate exhibited the maximum decolorization of $83 \%$, followed by $64 \%$ by RS1 and $57 \%$ by RS3. As the RS2 isolate exhibited the highest decolorization potential, it was further identified and used for melanoidin decolorization. Thus, RS2 was used alone for further studies. 


\section{Identification of the selected fungal isolate}

The potential isolate RS2 was identified as Aspergillus brasiliensis by using the BIOLOG method for the identification of fungi (Frąc, 2012; Pertile et al., 2020). The Biolog FF MicroPlate ${ }^{\mathrm{TM}}$ revealed the characteristic "fingerprint" of the RS2 isolate, which was matched in the MicroLog ${ }^{\mathrm{TM}}$ software, revealing it to be $A$. brasiliensis. The macroscopic and microscopic identification of the RS2 isolate using the FF database photo library confirmed the fungal isolate to be $A$. brasiliensis.

\section{Effect of the temperature on the growth of the RS2 isolate}

The effect of different temperatures, viz $5-45^{\circ} \mathrm{C}$, was evaluated for melanoidin decolorization by the RS2 strain. The maximum decolorization was observed for $A$. brasiliensis at $37^{\circ} \mathrm{C}$, where the largest recorded zone of clearance was $6.4 \mathrm{~cm}$. A further increase in temperature led to a decrease in the decolorization efficiency of the strain (Fig. 1).

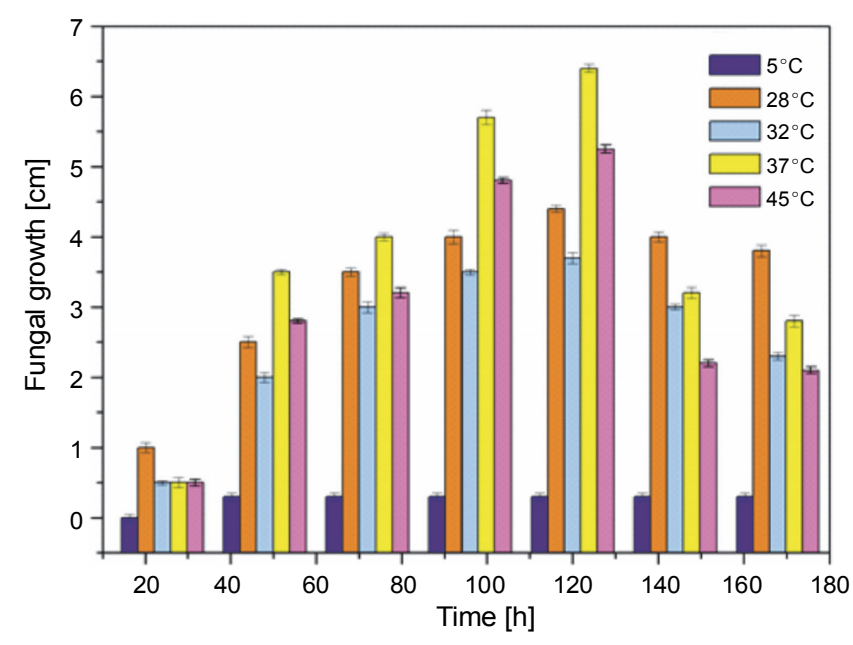

Fig. 1. Effect of different temperatures and time periods on the growth of $A$. brasiliensis

\section{Effect of different incubation periods on melanoidin decolorization}

Immediately after the optimization of the temperature for melanoidin decolorization by RS2 in a liquid medium, the best incubation period for decolorization was determined. The maximum decolorizationzone of $6.4 \mathrm{~cm}$ was observed at $120 \mathrm{~h}$ of incubation. A further increase in the incubation period did not increase the decolorization level (Fig. 1).

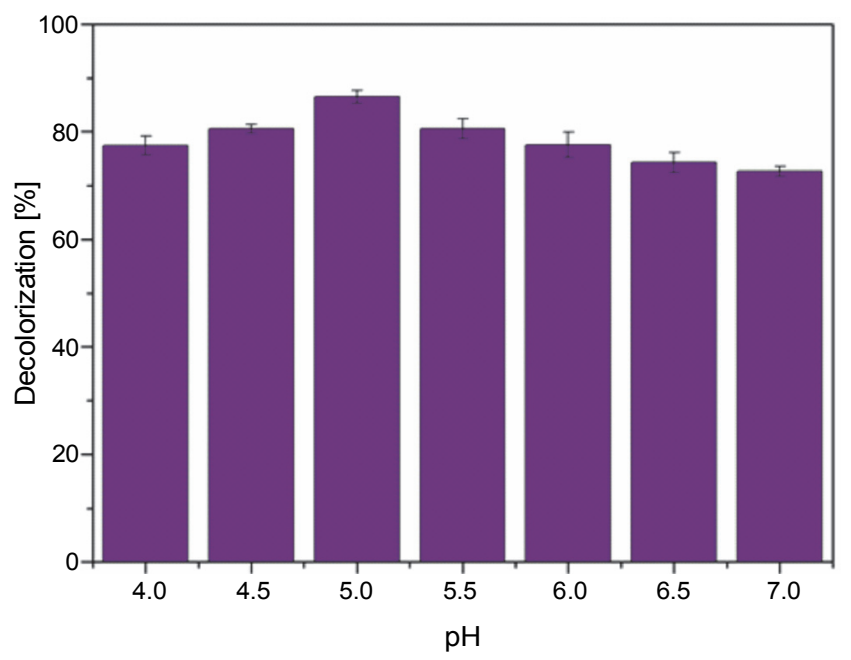

Fig. 2. Effect of $\mathrm{pH}$ on the decolorization of melanoidin by $A$. brasiliensis

\section{Effect of pH on melanoidin decolorization by the selected fungal strain}

Different $\mathrm{pH}$ values of the basal medium, viz. 4.0-7.0, were evaluated for melanoidin decolorization by the fungal strain at optimal temperature and incubation periods. A. brasiliensis showed the highest decolorization of $86.5 \%$ at $\mathrm{pH}$ 5. Further, an increase in $\mathrm{pH}$ to neutral reduced the decolorization level to $72.6 \%$ (Fig. 2).

\section{Effect of different carbon sources on melanoidin decolorization}

Carbon sources play a vital role in the growth of fungi, which contributes to the decolorization of the melanoidin pigment. Different carbon sources, namely starch, lactose, sucrose, dextrose, and maltose, at $2 \%$ concentration were individually tested in the basal medium at the optimum temperature, incubation period, and $\mathrm{pH}$ for the fungal isolate to observe the effect on melanoidin decolorization. The maximum decolorization of $87.43 \%$ was observed when starch was used as the carbon source, followed by lactose (76.87\%) and sucrose (73.36\%) which also showed good results. Maltose (64.12\%) and dextrose (62.27\%) exhibited the smallest decolorization of effluent (Fig. 3).

\section{Effect of different concentrations of starch on the decolorization potential of the fungal isolate}

In another set of the experiments, different concentrations of starch $(0.5$ to $3.0 \%)$ in the medium were tes- 


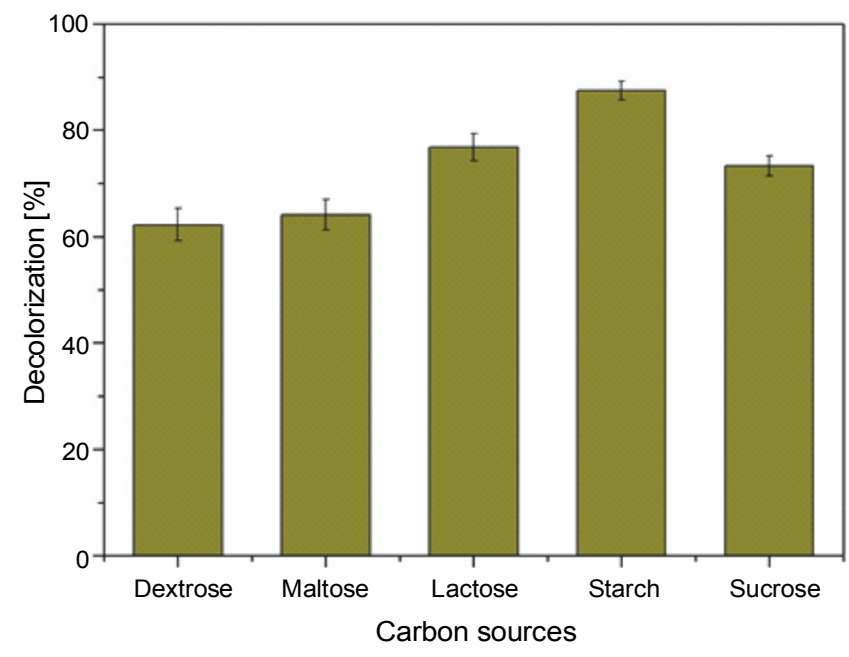

Fig. 3. Effect of different carbon sources on the decolorization of melanoidin by $A$. brasiliensis

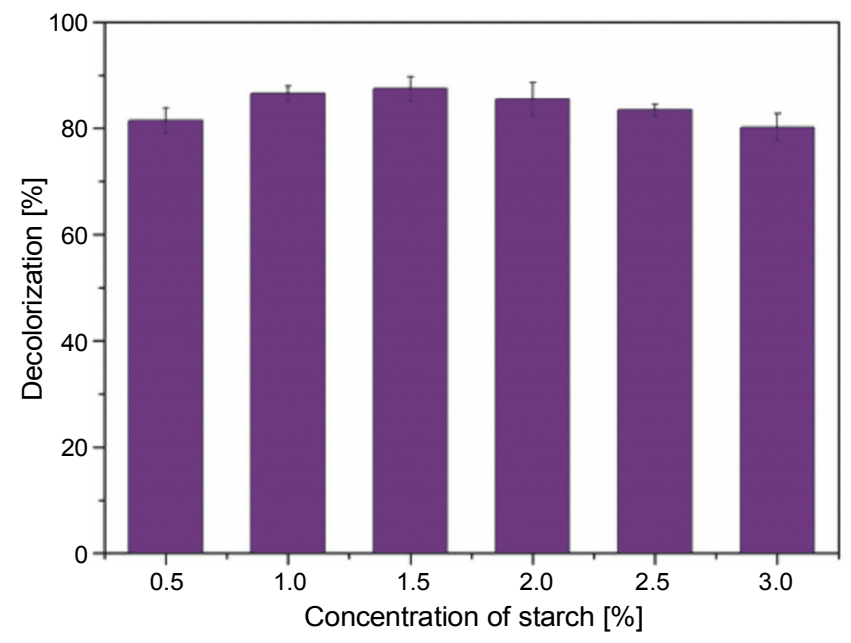

Fig. 4. Effect of different concentrations of starch on the decolorization of melanoidin by $A$. brasiliensis

ted for the ability of RS2 to decolorize melanoidin under the same growth conditions in which carbon sources were evaluated. The maximum decolorization of $87.45 \%$ was observed at $1.5 \%$ starch concentration. A later study showed that decolorization decreased above and below these levels (Fig. 4).

\section{Effect of different nitrogen sources on melanoidin decolorization}

Fungal growth is significantly affected by nitrogen sources. Various nitrogen sources, namely beef extract, peptone, yeast extract, sodium chloride and ammonium sulfate, were tested to evaluate their effect on melanoidin decolorization by the RS2 isolate. The nitrogen sources were added at the concentration of $1.5 \%$ to the basal medium for the decolorization experiment. The addition

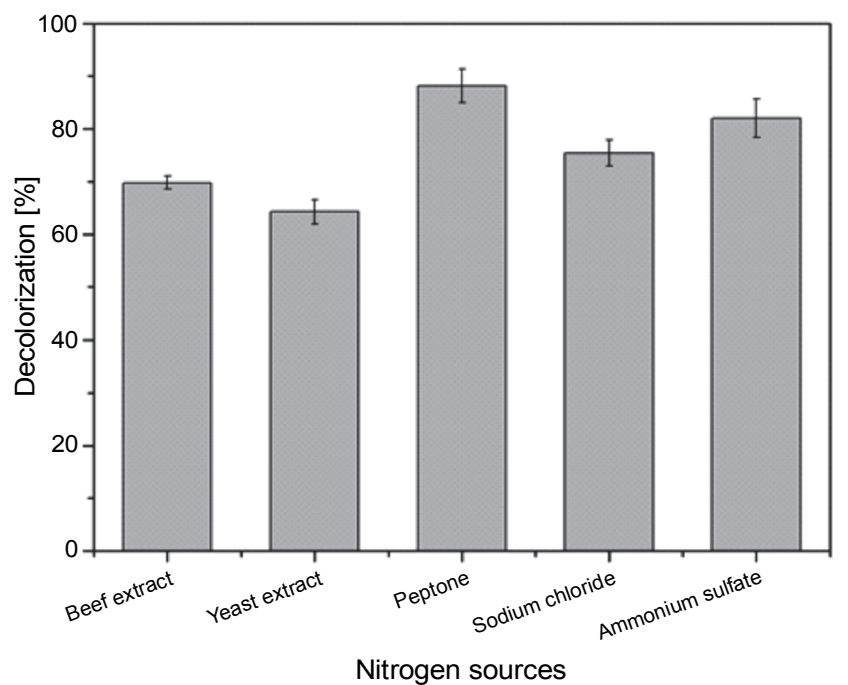

Fig. 5. Effect of different nitrogen sources on the decolorization of melanoidin by $A$. brasiliensis

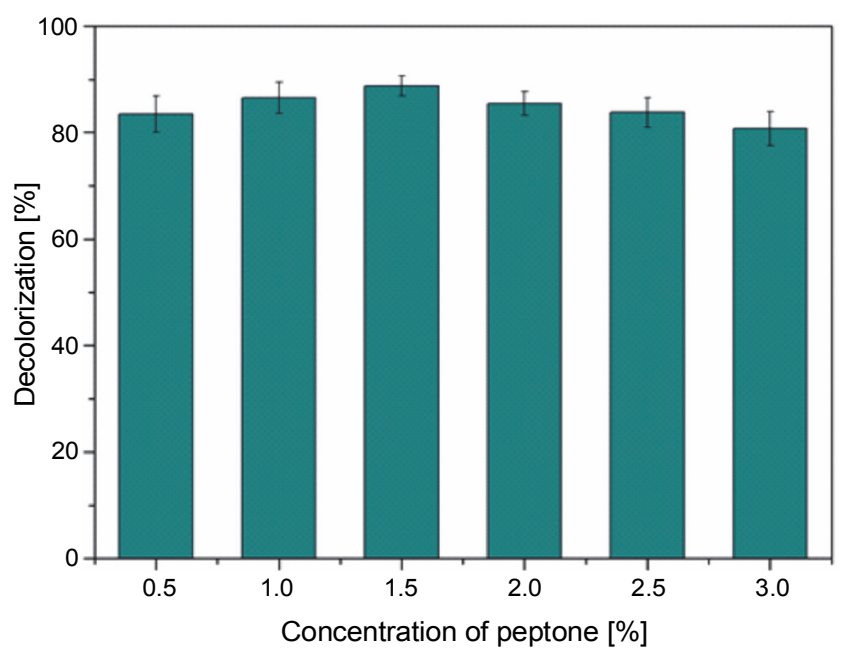

Fig. 6. Effect of different concentrations of peptone on the decolorization of melanoidin by $A$. brasiliensis

of peptone led to the maximum decolorization yield of $88.17 \%$. Furthermore, the addition of ammonium sulfate yielded a good decolorization level (81.27\%). All other nitrogen sources resulted in an effective decolorization of the melanoidin pigment, but it was still lower than that of peptone (Fig. 5). Decolorization was $69.8 \%$ and $75.4 \%$ with the addition of beef extract and sodium chloride, respectively.

\section{Effect of different concentrations of peptone on the decolorization potential of the fungal isolate}

To optimize the effect of peptone on the decolorization potential of RS2, its different concentrations $(0.5$, $1.0,1.5,2.0,2.5$, and $3.0 \%$ ) were added to the medium. The remaining conditions were the same as those used 
for nitrogen source evaluation. $A$. brasiliensis showed the best decolorization result $(88.74 \%)$ at $1.5 \%$ peptone concentration in the medium. A further increase in the concentration of peptone reduced the decolorization potential of $A$. brasiliensis. At $3 \%$ peptone concentration, 80\% melanoidin decolorization was observed (Fig. 6).

\section{Discussion}

In the present study, three fungal strains (RS1, RS2, and RS3) were tested for their decolorization potential of the melanoidin pigment in the distillery effluent from soil and molasses wastewater. Among these strains, RS2 showed the best results and was further used in the study. This fungus was identified as $A$. brasiliensis at the CMDR using the BIOLOG method for fungus identification. The decolorization potential of the fungal isolate depends on its ability to utilize the contaminants as its source of nutrition. Melanoidin shows antioxidant activity, which kills the microbial flora around it. Only a selective group of fungi can survive in such conditions and utilize melanoidin as a nutrient source (Wang et al., 2011).

In the present study, it was observed that $A$. brasiliensis could tolerate temperature upto $37^{\circ} \mathrm{C}$ without affecting its exponential growth phase during which melanoidin decolorization occurs (Fig. 1). Cetin and Donmez (2006) observed that the decolorization activity was suppressed at $45^{\circ} \mathrm{C}$ in mixed cultures of Alcaligenes faecalis and Comomonas acidovorans. This effect might be due to the loss of cell viability or deactivation of the enzymes responsible for decolorization at higher temperatures. Some researchers reported that melanoidin decolorization by Aspergillus sp. and Pseudomonas was due to the activity of their enzymes such as oxidase, ligninase, and peroxidase (Jadhav et al., 2011). Mohana et al. (2007) reported that increasing the temperature from 20 to $37^{\circ} \mathrm{C}$ increased the decolorization potential, and a further increase in the temperature to above $40^{\circ} \mathrm{C}$ decreased the decolorization ability of a microbial consortium of Pseudomonas aeruginosa, Stenotrophomonas, and Proteus. Thus, it is suggested that the optimal temperature for melanoidin decolorization depends on the variation of microbial strains and their genetic diversity as they are isolated from a very wide range of climatic conditions.

Various authors have reported that melanoidin decolonization by different microorganisms require varying incubation periods. In the present study, the maximum decolorization by $A$. brasiliensis was observed at $120 \mathrm{~h}$ of incubation (Fig. 1). Mohana et al. (2007) reported decolorization of effluent using a consortium of $P$. aeruginosa, Stenotrophomonas, and Proteus required an incubation period of $96 \mathrm{~h}$ as bacteria are fast growers than fungi. Jadhav et al. (2011) also reported that the maximum decolorization of melanoidin required an incubation period of $48 \mathrm{~h}$ due to a high enzyme production by $P$. aeruginosa in the medium.

The concentration of hydrogen ions in the medium (measured as $\mathrm{pH}$ value) is another important parameter that is directly related to the growth and activity of microorganisms. In the present study, the maximum decolorization of $86.5 \%$ by $A$. brasiliensis was observed at pH 5.0 (Fig. 2). This might be because every microorganism requires a specific $\mathrm{pH}$ for its growth and production of enzymes (Chandrakant and Kedar, 2013).

Various carbon sources were used in the study to test their influence onthe decolorization process. The maximum decolorization (87.45\%) was observed when starch was used at $1.5 \%$ concentration in the medium (Fig. 4). This may be due to the higher uptake of starch by the fungal isolate compared to the other carbon sources tested. Raghukumar and Rivonkar (2001) also observed the highest decolorization when starch and glucose were utilized as carbon sources by the white rot fungus Flavodon flavus. A further increment in starch concentration reduced the yield of melanoidin decolorization. Importantly, in the present study, it was observed that low concentrations of starch reduced the growth of $A$. brasiliensis, causing an overallreduction in the decolorization yield.

Finally, different nitrogen sources were optimized and tested for melanoidin decolorization potential. Among the different nitrogen sources tested in the present study, the highest melanoidin decolorization was reported when peptone at $1.5 \%$ concentration was added to the medium (Fig. 5 and Fig. 6). Similarly, various nitrogen sources were optimized by different researchers while testing melanoidin decolorization potential, but peptone was found to be the most effective nitrogen source for color removal (Tiwari et al., 2012). It was observed that at a higher nitrogen concentration of $3 \%$, the decolorization process was reduced due to surplus supplementation of nitrogen, which inhibited the growth of Coriolus hirsutus (Miyata et al., 2000; Angayarkanni et al., 2003). 
In the present study, $A$. brasiliensis was found to be a potential candidate for the bioremediation of melanoidin. Different fungal, bacterial, and yeast species have been tested for the remediation of melanoidin, but a complete removal of melanoidin has not yet been reported. A. brasiliensis decolorized melanoidin almost completely. We hope that this potential of the isolate is exploited at a larger industrial scale in the future for the bioremediation of melanoidin.

\section{Conclusions}

From the current study, it can be concluded that the treatment of distillery effluent with $A$. brasiliensis may be a cost-effective and environmentally friendly technology for the removal of the melanoidin pigment. The A. brasiliensis isolate exhibited a good potential to decolorize melanoidin and can be utilized at the industrial scale to resolve the problem of spent wash treatment. The bioremediation of melanoidin using $A$. brasiliensis is cheap, as the fungus grows well even at low concentrations of carbon and nitrogen sources and doesnot have fastidious nutrition requirement. A genetic improvement of the identified strain can be explored in the future to further improve its decolorization efficiency. The high decolorization percentage in the presence of starch as the carbon source and peptone as the nitrogen source suggests that the process can be performed economically without the involvement of any specialized medium. Future studies should focus on the utilization of $A$. brasiliensis for the decolorization of melanoidin at a larger scale and optimization of the parameters of decolorization. The isolate can be potentially exploited at the industrial scale for the bioremediation of melanoidin, and it may prove to be an asset in reducing environmental degradation caused by this contaminant.

\section{Acknowledgments}

The authors would like to thank the Choithram College of Professional Studies, Indore, M.P., India, and St. Aloysius College, Jabalpur, M.P., India, for providing the necessary facilities to conduct the research work. PKP is grateful to the University Grant Commission, New Delhi, India, for providing financial assistance (UGC-DSKPDF No.F.4-2/2006 BSR/BL/ 17-18/0442).

\section{References}

Angayarkanni J., Palaniswamy M., Swaminathan K. (2003) Biotreatment of distillery effluent using Aspergilus niveus. Bull. Env. Contamin. Toxicol. 70: 268-277.
Bernardo E.C., Egashitra R., Kawasaki J. (1997) Decolorization of molasses wastewater using activated carbon prepared from cane bagasse. Carbon 35: 1217-1221.

Cetin D., Donmez G. (2006) Decolorization of reactive dyes by mixed cultures isolated from textile effluent under anaerobic conditions. Enzym. Microbial. Technol. 38: 926-930.

Chandra R., Bhargava R.N., Rai V. (2008) Melanoidin as major colourant in sugarcane molasses based distillery effluent and its degradation. Biores. Technol. 99: 4648-4660.

Chandrakant M., Kedar. (2013) Physio-chemical analysis and microbial degredation of spent wash from sugar industries. Res. J. Chem. Sci. 3: 53-56.

Fito J., Alemu K. (2019) Microalgae-bacteria consortium treatment technology for municipal wastewater management. Nanitechnol. Environ. Eng. 4: 1-9.

Frąc M. (2012) Mycological evaluation of diary sewage sludge and its influence on functional diversity of soil microorganisms. Acta Agrophys. Monographiae. Inst. Agrophys. PAS, Lublin, Poland.

Jadhav J.P., Phugare S.S., Dhanve R.S., Jadhav S.B. (2011) Rapid biodegradation and decolorization of direct Orange 39 by an isolated bacterium Pseudomonas aeruginosa strain BCH. Biodegradation 21: 453-463.

Karnwal A. (2019) Textile azo dye decolorization and detoxification using bacteria isolated from textile effluents. BioTechnologia 100(4): 373-385.

Kumar V., Wati L., Nigam P., Banat I.M., Yadav B.S., Singh D., Merchant R. (1998) Decolorization and biodegradation of anaerobically digested sugarcane molasses spent wash effluent from biomethanation plants by white rot fungi. Proc. Biochem. 33: 83-88.

Miyata N., Mori T., Iwahori K., Fujita M. (2000) Microbial decolorization of melanoidin containing wastewater: Combined use of activated sludge and the fungus Coriolushirsutus. J. Biosci. Bioeng. 89: 145-150.

Mohana S., Desai C., Datta M. (2007) Biodegradation and decolorization of anaerobically treatment distillery effluent spent-wash by a novel bacterial consortium. Biores. Technol. 98: 333-339.

Moosvi S., Keharia H., Madamwar D. (2005) Decolorization of textile dye reactive violet by a newly isolated bacterial consortium RVM 11.1. World J. Microbiol. Biotechnol. 21: 667-672.

Naik N., Jagdeesh K.S., Noolvi M.N. (2010) Enhanced degradation of melanoidin and caramel in bio methanated distillery spent wash by microorganisms isolated from mangroves. Iranica J. Ener. Environ. 1: 347-351.

Pant D., Adholeya A. (2007) Biological approaches for treatment of distillery wastewater: a review. Biores. Technol. 98: 2321-2334.

Pertile G., Frac M., Formal E., Oszust K., Gryta A., Yaguchi T. (2020) Molecular and metabolic strategies for postharvest detection of heat resistant fungus Neosartorya fischeri and its discrimination from Aspergillus fumigatus. Postharvest Biol. Tec. 161: 11082.

Raghukumar C., Rivonkar G. (2001) Decolorizaiton of molasses spent wash by the white-rot fungus Flavodon flavusi- 
solated from a marine habitat. Appl. Microbiol. Biotech. 55: 510-514.

Sharma P., Singh T.A., Bharat B., Bhasin S., Modi H.A. (2018) Approach towards different fermentative techniques for the production of bioactive actinobacterial melanin. BeniSuef Univ. J. Basic Appl. Sci. 7: 695-700.

Subramanian K.A., Singhal S.K., Saxena M., Singhal S. (2005) Utilization of liquid biofuels in automatic diesel engines: an Indian perspective. Biomass Bioenergy 29: 65-72.

Tiwari P.K., Batra V.S., Balakrishnan M. (2007) Water management initiatives in sugarcane molasses based distilleries in India. Res. Conserv. Recycl. 52: 351-367.
Tiwari S., Gaur R., Singh R. (2012) Decolorization of recalcitrant organic compound (Melanoidin) by a novel thermotolerant yeast, Candida tropicalis RG-9. BMC Biotechnol. 12: 30-37.

Vasudev K., Kuhad R.C. (1994) Decolorization of polyR-478 (polyvinylamine sulfonate anthrapyridone) by Cyathus bulleri. Folia Microbiol. 39: 61-64.

Wang H., Qian H., Yao W. (2011) Melanoidins produced by the Maillard reaction: structure and biological activity. Food Chem. 28: 573-584. 